\section{Foods Eaten by the Rocky Mountain Elk}

\section{ROLAND C. KUFELD}

Highlight: Forty-eight food habits studies were combined to determine what plants are normally eaten by Rocky Mountain elk (Cervus canadensis nelsoni), and the relative value of these plants from a manager's viewpoint based on the response elk have exhibited toward them. Plant species are classified as highly valuable, valuable, or least valuable on the basis of their contribution to the diet in food habits studies where they were recorded. A total of 159 forbs, 59 grasses, and 95 shrubs are listed as elk forage and categorized according to relative value.

Knowledge of the relative forage value of plants eaten by elk is basic to clk rangc surveys, and to planning and evaluation of habitat improvement programs. Numerous elk food habits studies have been conducted; however, individual studies are limited to a specific area, and relatively few plant species are found in the diet compared to the number of plants eaten by elk throughout their range. The amount of a particular species consumed in one study may or may not be indicative of its true value as elk forage. The purpose of this inquiry is to combine all food habits work to determine which plants are eaten by elk, and their relative value as reflected by the degree to which they are normally sought.

\section{Methods}

With one exception only studies which pertain to food habits of the Rocky Mountain elk (Cervus canadensis nelsoni) in the western U. S. and Canada were included. The exception study involving C. canadensis manitobensis (Blood, 1966) was incorporated because of its quality and because the plants eaten closely paralleled those consumed on elk ranges in Montana and Idaho. Studies of Rocky Mountain elk transplanted to areas outside their normal range were excluded.

An extensive literature review was made to assemble references concerning

The author is wildlife researcher, Colorado Division of Wildlife, Fort Collins.

The paper is a contribution of Federal Aid to Wild life Restoration Project W-101-R.

Acknowledgment is made to Mrs. Page Smith and to the Library Reference Service, Fed. Aid in Fish and Wildl. Res., Denver, Colo., for assistance in assembling elk food habits references; and to Dr. Lee E. Yeager for reviewing the manuscript.

Manuscript received May 19, 1972. elk food habits, and studies meeting the following criteria were incorporated: (1) Data must be original and derived from a specific effort to collect food habits information. References containing statements of what elk eat based on general knowledge, or those which summarized previous food habits studies were excluded. (2) Data must be listed by species eaten, and relative quantity consumed must be expressed in terms which would allow degree of use to be categorized. (3) Season of use must be shown. (4) Data must be listed separately for elk. Studies which referred to combined deer and elk use or "game use" were excluded. (5) Studies with a very limited sample (for example, only two or three stomachs) were excluded. (6) Elk must have had cluded some pen feeding studies. (7) Study animals must not be starving. Forty-eight studies were incorporated in this summary.

Methods of data collection were assigned four categories: stomach analysis, feeding observations on wild animals, apparent use of plants, and pen feeding studies designed to determine preference. method of data collection and presentation; in number, relative abundance, availability, and relative palatability of plant species encountered; and in number of animals using the study area. Thus, firm guidelines cannot be established for comparing relative forage preference among several food habits studies. In every study, however, some plants were consumed more extensively than others. The procedure used herein involved categorizing plants encountered in cach study according to whether they were used (1) lightly (2) moderately or (3) heavily in relation to all species consumed in the particular reference. Plants which contributed less than $1 \%$ of the diet or which free choice of available foragc. This ex-

Studies of food habits differ widely in were reported as trace amounts were excluded. Factors such as relative plant abundance in relation to consumption were considered in assigning plants to use categories when such information was available. An average ranking for each species was then determined on the basis of all studies where it was found to contribute at least $1 \%$ of the diet.

The following terminology is used throughout this report. Highly valuable plant-One avidly sought by elk and which made up a major part of the diet in food habits studies where encountered, or which was consumed far in excess of its vegetative composition. These had an average ranking of 2.25 to 3.00 . Valuable plants-One sought and readily eaten but to a lesser extent than highly valuable plants. Such plants made up a moderate part of the diet in food habits studies where encountered. Valuable plants had an average ranking of 1.50 to 2.24. Least valuable plant-One eaten by elk but which usually made up a minor part of the diet in studies where encountered, or which was consumed in a much smaller proportion than it occurred on the range. Least valuable plants had an average ranking of 1.00 to 1.49 . These terms are used to reflect the relative value of a plant's presence on elk range from a manager's viewpoint because of the response elk have exhibited toward it. Value as used here does not consider nutrient quality or the importance of a species in maintaining a certain desired stage of ecological succession.

Data were separated by the following seasons of use: Winter-December, January, February; Spring-March, April, May; Summer-June, July, August; FallSeptember, October, November.

\section{Results}

\section{Seasonal Use of Major Forage Groups}

Winter-Winter use is concentrated on either grasses or shrubs, depending on forage availability. The following authors reported winter grass consumption on Montana's predominately grass ranges as varying from 63 to $100 \%$, and averaging 84\%: Casagranda and Janson (1957); Constan (1967); Gordon (1968); Greer (1959); Greer et al. (1970); and Morris and Schwartz (1957). Winter shrub use averaged $9 \%$ and forb use $8 \%$ in these studies.

DeNio (1938) reported 65\% winter use of grasses, $15 \%$ shrubs, $2 \%$ forbs, and $5 \%$ mosses and lichens in Montana, northern Idaho, and northeastern Washington. Winter grass consumption was as high as 97\% in Jasper Park, Alta. (Cowan, 1947). Shrubs comprised $95 \%$ of the winter diet in New Mexico (Lang, 1958), 62\% in Manitoba (Blood, 1966), and $82 \%$ in 
Idaho of which $22 \%$ were conifers (Trout and Leege, 1971). Boyd (1970) recorded $57 \%$ shrub use in Colorado from December through April. In these studies grass consumption averaged $22 \%$ and forbs were eaten in only two studies where maximum consumption was $10 \%$.

Spring-Spring grass use on eight Montana studies remained high, averaging 87\% (Eustace, 1967; Greer et al., 1970; Gordon, 1968; Kirsch, 1963; Mackie, 1970; Morris and Schwartz, 1957; Rouse, 1957; and Stevens, 1966). Little information outside Montana was available on spring use of major forage classes except for Manitoba, where use of grasses, shrubs, and forbs was 54, 37 and 9\%, respectively,

Summer-Forbs became important forage during summer. The summer diet in Montana averaged $64 \%$ forbs, $30 \%$ grasses, and 6\% shrubs (Eustace, 1967; Greer et al., 1970; Kirsch, 1963; Mackie, 1970; Morris and Schwartz, 1957; Rouse, 1958; Stevens, 1966). Rouse (1958) recorded $100 \%$ forbs in the summer diet. Summer forage consumption in Colorado, as reported by Nichols (1957), was 58\% grasses, $41 \%$ forbs, and 1\% shrubs. Boyd (1970), also in Colorado, recorded 78\% summer use of grasses, $12 \%$ forbs, and $10 \%$ shrubs. Studies where high summer shrub use were recorded were made by Young and Robinette (1939) in Idaho, where use was $55 \%$ shrubs, $25 \%$ grasses, and $20 \%$ forbs; and by Blood (1966), in Manitoba, who noted $52 \%$ use of shrubs, $22 \%$ grasses, and $26 \%$ forbs.

Fall-Primary use reverts to grasses in the fall in Montana where grass use averaged $73 \%$ in nine studies (Greer, 1959; Greer, 1960; Greer et al., 197.0; Kirsch, 1963; Mackie, 1970; Morris and
Schwartz, 1957; Peek 1963; Rouse, 1957, Rush, 1932). High grass use in fall was also found in Colorado by Boyd (1970), who recorded $92 \%$ grass consumed. In New Mexico, Burt and Gates (1959) found that grass comprised $84 \%$ of the fall diet; however, Lang (1958), also in New Mexico, recorded 77\% use of shrubs, $21 \%$ grasses, and $2 \%$ forbs. Shrub use was high in Manitoba and Idaho, where Blood (1955) and Young and Robinette (1939) found 55 and $40 \%$ use of shrubs, 37 and $40 \%$ use of grasses and 8 and $20 \%$ forb use, respectively.

\section{Plant Species Value}

Plant species eaten by elk and their relative value rankings for each season are listed by forbs in Table 1, grasses in Table 2 , and shrubs in Table 3 . Validity of these rankings increases with the number of references on which a ranking is based.

Table 1. Relative value of forb species eaten by Rocky Mountain elk.

\begin{tabular}{|c|c|c|c|c|c|}
\hline \multirow[b]{2}{*}{ Plant name } & \multicolumn{4}{|c|}{ Forage value $^{1}$} & \multirow[b]{2}{*}{ References $^{2}$} \\
\hline & Winter & Spring & Summer & Fall & \\
\hline Achillea & & & & $1.00-2$ & 29,36 \\
\hline Achillea millefolium & $1.33-3$ & & $1.00-4$ & $1.00-2$ & $9,14,17,18,30,31,37,44$ \\
\hline Actaea spicata & & & $1.00-1$ & $2.00+1$ & 48 \\
\hline Agastache urticifolia & & & $1.50+2$ & & 37,48 \\
\hline Agoseris glauca & & $1.50+4$ & $2.33 * 6$ & & $7,17,29,30,39,44$ \\
\hline Alectoria fremontii & $1.00-1$ & & & & 13 \\
\hline Allium textile & & $2.00+1$ & & & 32 \\
\hline Angelica lyallii & & & $1.00-1$ & $1.00-1$ & 48 \\
\hline Antennaria & $1.00-3$ & $1.00-2$ & $1.00-1$ & $1.00-2$ & $18,25,36,39$ \\
\hline Antennaria parvifolia & & $1.00-1$ & & & 44 \\
\hline Antennaria rosea & $1.00-1$ & & & & 18 \\
\hline Aquilegia flavesens & & $1.00-1$ & $1.00-1$ & & 7 \\
\hline Arenaria & & & $1.00-1$ & & 10 \\
\hline Arnica & & $1.50+2$ & $2.00+1$ & $1.00-1$ & $25,30,44$ \\
\hline Arnica cordifolia & & & $2.00+2$ & & 17,29 \\
\hline Arnica latifolia & & & $2.00+1$ & $3.00 * 1$ & 48 \\
\hline Arnica sororia & & $2.00+1$ & & & 32 \\
\hline Artemisia dracunculus & $2.00+1$ & & & & 42 \\
\hline Artemisia frigida & $1.33-6$ & $1.00-4$ & & & $14,20,24,25,29,30,42,44$ \\
\hline Artemisia longifolia & $1.00-1$ & & & $1.00-1$ & 32 \\
\hline Artemisia ludoviciana & $1.00-1$ & & & $2.00+1$ & 30,32 \\
\hline Asplenium felix-femina & & & $3.00 * 1$ & $2.00+1$ & 48 \\
\hline Aster & $2.00+2$ & $2.00+2$ & $1.75+4$ & $1.50+8$ & $18,21,23,24,25,29,30,33,36,39,42,43$ \\
\hline Aster canescens & & & $2.00+1$ & $3.00 * 1$ & 48 \\
\hline Aster commutatus & & & & $2.00+1$ & 32 \\
\hline Aster eatoni & & & $2.00+1$ & $3.00 * 1$ & 48 \\
\hline Astragalus & $1.50+2$ & & $3.00 * 1$ & $2.00+1$ & $30,42,48$ \\
\hline Astragalus miser & & & $1.00-1$ & & 30 \\
\hline Balsamorhiza saggittata & $1.75+4$ & $2.00+2$ & $1.00-1$ & & $3,7,11,18,38,42$ \\
\hline Boykinia heucheriformis & & & $3.00 * 1$ & $2.00+1$ & 48 \\
\hline Caltha leptosepala & & & $2.50 * 2$ & & 6,35 \\
\hline Castilleja & & & $1.00-1$ & & 37 \\
\hline Castilleja miniata & & & $1.00-1$ & & 48 \\
\hline Cerastium arvense & & $1.00-1$ & & & 44 \\
\hline Chalachortus elegans & & & $1.00-1$ & & 48 \\
\hline Chamaenerion angustifolium & $3.00 * 1$ & & $3.00 * 1$ & $2.00+1$ & 18,48 \\
\hline Cirsium & $1.00-1$ & $1.00-2$ & & $2.00+3$ & $20,21,25,36,42$ \\
\hline Cirsium foliosum & & $1.00-1$ & $1.00-1$ & $3.00 * 1$ & 30 \\
\hline Claytonia asarifolia & & & $3.00 * 1$ & $3.00 * 1$ & 48 \\
\hline Claytonia sibirica & & & $3.00 * 1$ & $3.00 * 1$ & 48 \\
\hline Clintonia uniflora & & & $1.00-1$ & $1.00-1$ & 48 \\
\hline Commandra sp & & & & $1.00-1$ & 25 \\
\hline Commandra pallida & & & $2.00+1$ & & 33 \\
\hline
\end{tabular}


Coptis occidentalis

Corydalis scouleri

Delphinium sp

Delphinium bicolor

Delphinium scopulorum

Dodecatheon conjugens

Dodechatheon pauciflorum

Dodocathelon conjugens

Epilobium angustifolium

Equisetum

Erigeron

Eriogonum

Eriogonum heracleoides

Eriogonum umbellatum

Fragaria

Fragaria virginiana

Fraseria sp.

Gaillardia sp.

Galium boreale

Geranium

Geranium richardsonii

Geranium viscossissimum

Geum

Geum triflorum

Geum turbinatum

Glycyrrhiza lepidota

Hedysarum sulphurescens

Helianthus maximilliana

Heracleum lanatum

Hieracium sp.

Hieracium albiflorum

Hieracium chapacanum

Hieracium cynoglossoides

Hieracium scouleri

Heuchera glabella

Hydrophyllum capitatum

I ris missouriensis

Lactuca pulchella

Lactuca serriola

Lathyrus

Lathyrus laetivirens

Ledum groenlandicum

Liatris punctata

Ligusticum grayi

Ligusticum scopulorum

Ligisticum tenufolium

Lithospermum ruderale

Lupinus

Lupinus leucophyllus

Lupinus ornatus

Lupinus sericeus

Medicago sativa

Melilotus officinalis

Mertensia ciliata

Microseris

Mitella stauropetala

Myosotis alpestris

Oenothera flava

Opuntia sp.

Oreoxis alpina

Osmorhiza occidentalis

Oxytropis

Oxytropis splendens

Oxytropis viscida

Pedicularis cystopteridifolia

Pedicularis groenlandica

Pedicularis racemosa

Penstemon

Penstemon confertus

Penstemon pinetorum

Penstemon procerus

Petasites saggittatus

Phacelia heterophylla

Phlox

Phlox hoodii

Polemonium delicatum

Polygonum phytolaccaefolium
$1.00-1$

$2.00+1$

$2.00+1$

$1.00-1$

$2.00+1$

$2.00+1$

$2.00+2$

$3.00 * 1$

$1.00-1$

$3.00 * 1$

$2.00+2$

$1.00-1$

$1.00-1$

$2.00+1$

$2.00+1$

$1.50+2$

$1.50+2$

$2.00+1$

$1.00-3$

$1.00-1$

$2.00+1$

$1.00-1$

$1.00-1$

$2.00+1$

$1.50+2$

$3.00 * 1$

$2.00+2$

$2.43 * 7$

$2.00+1$

$1.00-1$

$2.00+1$

$1.00-1$

$3.00 * 1$

$2.00 * 1$

$1.00-1$

$1.00-2$

$2.00+1$

$2.00+1$

$2.00+1$

$1.00-1 \quad 1.00-1$

$1.00-1$

$2.00+1$

$3.00 * 1$

$2.00+1$

$1.00-1$

$2.00+$

$2.00+1$

$2.00+1$

$2.00+1$

$1.00-1$

$1.00-2$

$1.50+2$

$2.00+$

$+1$

$3.00 *$

$2.00+1$

$2.67 * 3$

$1.00-1$

$2.00 * 2 \quad 2.00+1$

$3.00 * 1$

$1.00-1 \quad 2.50 * 2$

$3.00 * 1$

$1.00-1 \quad 1.00-2$

$1.00-1$

$3.00 * 1$

$3.00 * 1$

$3.00 * 1$

$3.00 * 2$

$1.00-1$

$1.00-1$

$3.00 * 1$

$2.00+1$

$2.00+1$

$2.00+1$

$1.00-1$

$1.00-1$
$1.00-1$
$1.00-1$

$3.00 * 1$

$1.00-1$

$3.00 * 1$

$3.00 * 1$

$3.00 * 1$

$2.00+1$

$3.00 * 1$

$2.00+1$

$2.00+1$

$1.50+2$

$1.00-1$

$1.00-1$

$1.00-2$

$1.00-1$

$2.00+2$

$3.00 * 1$

$2.00+1$

$1.00-1$

$2.00+1$

$1.00-1$

$3.00 * 1$

$1.00-1$

$1.00-1$

$2.40 * 5$

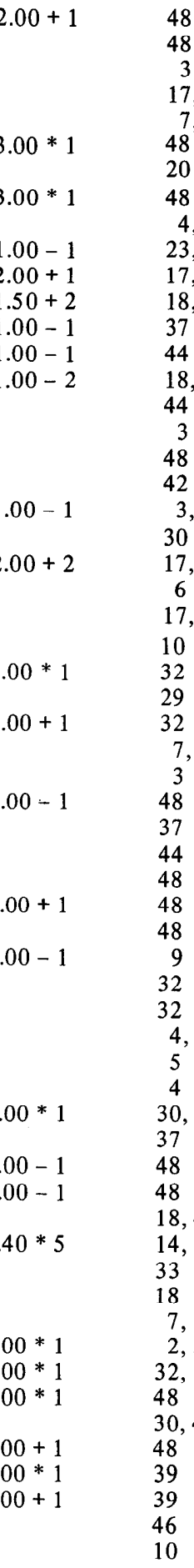

$1.00-1$
$2.00+1$

8

17,20

7,48

20

4,29

23,25

$18,24,25$

37

44

$18,25,30,48$

44

48

42

$3,25,29,30$

30

$17,29,30,39,40,44,48$

17,20

10

29

7,48

3
48

37

44

48

48

9

4,6

0,42

37

8,42

, 17, 23, 24, 25, 29, 36, 37, 39, 42, 44, 48

$7,20,30,42$

2,33

32,33

48

0,44

48

46

10

37,48

25

29

30

17

48

$1.00-1 \quad 48$

48

$3.00 * 1 \quad 48$

37

4

$1.00-1 \quad 2.00+1 \quad 48$

$1.00-1 \quad 25,36$

39

$2.00+1 \quad 1.00-1 \quad 48$

$2.50 * 2 \quad 37,48$ 
Potentilla

Potentilla glandulosa

Potentilla gracilis

Pteris aquilina

Ranunculus

Ranunculus glaberrimus

Rumex paucifolius

Sanguisorba sitchensis

Selaginella densa

Senecio

Senecio columbianum

Senecio triangularis

Smilacina racemosa

Smilacina stellata

Solidago

Sonchus arvensis

Sphaeralcea rivularis

Stellaria

Taraxacum

Taraxacum officinale

Thermopsis montana

Thermopsis pinetorum

Tragopogon

Tragopogon dubius

Trifolium

Trifolium dasyphyllum

Trifolium haydeni

Trifolium repens

Trifolium rydbergi

Typha

Valeriana sitchensis

Veratrum eschscholtzii

Vicia americana

Viola nuttallii

Wyethia

Xanthium strumarium

Xerophyllum tenax

Zizia aptera

$\begin{array}{lll}1.00-2 & 1.29-7 & 1.50+2 \\ & 1.50+2 & 2.00+1 \\ & 1.00-1 & \\ & 1.00-1 & \\ & 2.00+1 & \\ 1.00-1 & 1.00-1 & \\ 2.00+1 & 2.00+1 & \\ & 2.00+1 & \\ 1.00-1 & & \\ & 1.00-2 & \\ & 3.00 * 1 & \\ & 2.50 * 2 & 3.00 * 1 \\ & & 2.00+1 \\ & & 2.00+1\end{array}$

$1.00-1$

$1.00-1$

$2.00+1$

$3.00 * 1$

$1.00-2 \quad 3.00 * 3$

$1.00-2 \quad 2.00+3$

$1.00-1$

$2.00+1$

$2.00+1 \quad 2.00+1$

$1.00-1 \quad 2.00+3$

$3.00 * 1$

$2.00+1 \quad 2.00+1$

$3.00 * 1$

$2.00+1 \quad 2.00+1$

$3.00 * 1$

$1.00-1$

$2.00+1 \quad 2.00+1$

$1.00-1 \quad 1.00-1$

$2.50 * 2$

$3.00 * 1$

$1.00-1$

$2.00+1$

$3.00 * 1 \quad 3.00 * 1$
$10,17,25,29,30,39,44$

37,48

29

48

30

7

7

44

$4,18,35,39$

48

37,48

48

48

18

4

30

$17,29,44$

$3.00 * 1$

$1.00-1$

$1.00-1$

$4,7,30$

39

9

21

$20,32,42$

$17,30,40$

10

7

44

$1.00-1$

$3.00 * 1$

48

48

4,32

7

$2.50 * 2$

$36,39,40$

32

18,48

30

${ }^{1}$ Each entry consists of 3 parts. The first number is the computed value ranking. The second part is the value ranking symbol: $-=1$ least valuable; $+=$ valuable; ${ }^{*}=$ highly valuable. The third part is the number of references upon which the ranking is based.

2 Numbers indicate references in literature cited section on which value rankings were based.

Table 2. Relative value of grass species eaten by Rocky Mountain elk.

\begin{tabular}{|c|c|c|c|c|c|}
\hline \multirow[b]{2}{*}{ Plant name } & \multicolumn{4}{|c|}{ Forage value ${ }^{1}$} & \multirow[b]{2}{*}{ References $^{2}$} \\
\hline & Winter & Spring & Summer & Fall & \\
\hline Agropyron cristatum & & $2.00+1$ & & & 29 \\
\hline Agropyron dasystachyum & & $1.00-1$ & & & 29 \\
\hline Agropyron pauciflorum & $3.00 * 1$ & & & & 18 \\
\hline Agropyron scribneri & & & $2.00+1$ & & 10 \\
\hline Agropyron smithii & $3.00 * 1$ & $3.00 * 1$ & $2.00+1$ & $3.00 * 1$ & 32 \\
\hline Agropyron spicatum & $3.00 * 8$ & $2.00+8$ & $1.50+2$ & $2.00+1$ & $8,11,14,17,18,20,29,30,33,38,42,43,44$ \\
\hline Agropyron subsecundum & & & $1.00-1$ & $3.00 * 1$ & 17,39 \\
\hline Agropyron trachycaulum & & & $1.00-1$ & $2.00+1$ & 39 \\
\hline Agrostis exarata & & & $3.00 * 1$ & $2.00+1$ & 48 \\
\hline Agrostis idahoensis & & & $1.00-1$ & & 35 \\
\hline Bromus & $1.00-1$ & & $1.67+3$ & & $17,30,44$ \\
\hline Bromus carinatus & & & $3.00 * 1$ & $3.00 \quad 1$ & 48 \\
\hline Bromus inermis & & & $2.00+2$ & $2.00+1$ & 29,33 \\
\hline Bromus marginatus & $3.00 * 1$ & & & & 18 \\
\hline Bromus tectorum & $2.00+2$ & & & & 8,13 \\
\hline Calamagrostis canadensis & $2.50 * 2$ & & & & 18,30 \\
\hline Calamagrostis rubescens & $2.00+2$ & $3.00 * 1$ & $3.00 * 1$ & $1.50+2$ & $18,30,48$ \\
\hline Camassia quamash & & $2.00+1$ & $1.00-1$ & & 30 \\
\hline Carex & $2.00+3$ & $1.00-1$ & $1.67+6$ & $2.33 * 3$ & $2,17,18,25,30,35,37,38,39,44$ \\
\hline Carex filifolia & $3.00 * 1$ & & & & 18 \\
\hline Carex geyeri & $2.33 * 3$ & $3.00 * 1$ & $2.33 * 3$ & $2.50 * 2$ & $13,18,29,30,37,48$ \\
\hline Carex nubicola & & $2.00+1$ & $2.00+1$ & & 7 \\
\hline Carex raynoldsii & & $2.00+1$ & $2.00+1$ & & 7 \\
\hline Danthonia & $2.00+1$ & $1.00-1$ & $2.00+1$ & & 30 \\
\hline Danthonia intermedia & $3.00 * 1$ & & & & 18 \\
\hline Danthonia parryi & $2.00+1$ & & & & 30 \\
\hline Danthonia unispicata & $2.00+1$ & & & & 11 \\
\hline Deschampsia caespitosa & & & $3.00 * 1$ & & 35 \\
\hline Distichlis stricta & $2.00+1$ & & & & 32 \\
\hline
\end{tabular}


Elymus flavescens

Elymus glaucus

Elymus innovatus

Festuca idahoensis

Festuca ovina

Festuca scabrella

Juncoides parviflorum

Juncus balticus

Juncus parryi

Koeleria cristata

Melica spectabilis

Orzyopsis hymenoides

Phleum

Phleum alpinum

Phleum pratense

Poa

Poa alpina

Poa canadensis

Poa compressa

Poa epilis

Poa secunda

Stipa

Stipa columbiana

Stipa viridula

Trisetum spicatum

Trisetum wolfii
Muhlenbergia

Muhlenbergia cuspidata

Sitanion hystrix

Stipa comata

\begin{tabular}{|c|c|c|c|c|}
\hline \multirow{3}{*}{$\begin{array}{l}3.00 * 1 \\
2.00+1\end{array}$} & $3.00 * 1$ & & & 46 \\
\hline & $2.00+1$ & $3.00 * 1$ & $3.00 * 1$ & 39,48 \\
\hline & & & $1.00-1$ & 39 \\
\hline \multirow[t]{2}{*}{$2.56 * 9$} & $2.83 * 6$ & $1.50+2$ & $3.00 * 2$ & $7,8,11,14,17,18,20,29,30,38,39,42,43,44$ \\
\hline & $2.00+1$ & $2.00+1$ & & 7 \\
\hline \multirow[t]{4}{*}{$2.40 * 5$} & $2.75 * 4$ & $1.00-2$ & $2.00+1$ & $11,17,18,20,29,30,42,44$ \\
\hline & & $3.00 * 1$ & $3.00 * 1$ & 48 \\
\hline & & $3.00 * 1$ & & 35 \\
\hline & & $3.00 * 1$ & & 37 \\
\hline \multirow[t]{2}{*}{$1.60+5$} & $1.50+4$ & $2.00+1$ & $2.00+2$ & $11,17,18,20,32,38,44$ \\
\hline & & $1.00-1$ & & 39 \\
\hline $1.50+2$ & & & & 11,38 \\
\hline \multirow[t]{4}{*}{$1.00-1$} & & & $2.00+1$ & 32 \\
\hline & & & $3.00 * 1$ & 46 \\
\hline & $2.00+1$ & $1.50+2$ & & 7,44 \\
\hline & $2.00+1$ & $2.00+2$ & & 7,35 \\
\hline $2.50 * 2$ & $2.00+1$ & $1.00-1$ & $3.00 * 1$ & 18,30 \\
\hline \multirow[t]{2}{*}{$2.00+5$} & $2.67 * 6$ & $2.00+5$ & $2.50 * 2$ & $13,14,17,20,29,30,33,42,44$ \\
\hline & & $2.00+1$ & & 30 \\
\hline $3.00 * 1$ & & & & 18 \\
\hline \multirow[t]{2}{*}{$3.00 * 1$} & $2.00+1$ & & $2.00+1$ & 18,32 \\
\hline & $2.00+1$ & $2.00+2$ & & 7,35 \\
\hline \multirow[t]{2}{*}{$2.00+1$} & $2.50 * 2$ & $1.50+2$ & $3.00 * 1$ & 7,32 \\
\hline & & $1.00-1$ & & 37 \\
\hline \multirow{3}{*}{$\begin{array}{l}2.33 * 3 \\
3.00 * 1\end{array}$} & $2.00+1$ & $2.00+1$ & $3.00 * 1$ & 11,3038 \\
\hline & & $1.00-1$ & & 18,37 \\
\hline & & & $3.00 * 1$ & 46 \\
\hline \multirow{2}{*}{$1.00-1$} & $2.00+1$ & & & 32 \\
\hline & & $\begin{array}{l}2.00+1 \\
2.00+1\end{array}$ & $2.00+1$ & $\begin{array}{l}17,29 \\
35\end{array}$ \\
\hline
\end{tabular}

1Each entry consists of 3 parts. The first number is the computed value ranking. The second part is the value ranking symbol: $-=$ least valuable; $+=$ valuable; $*=$ highly valuable. The third part is the number of references upon which the ranking is based.

2 Numbers indicate references in literature cited section on which value rankings were based.

Table 3. Relative value of shrub species eaten by Rocky Mountain elk.

\begin{tabular}{|c|c|c|c|c|c|}
\hline \multirow[b]{2}{*}{ Plant name } & \multicolumn{4}{|c|}{ Forage value ${ }^{1}$} & \multirow[b]{2}{*}{ References ${ }^{2}$} \\
\hline & Winter & Spring & Summer & Fall & \\
\hline Abies grandis & $1.00-2$ & & & & 18,45 \\
\hline Acer glabrum & $2.25 * 4$ & $1.00-1$ & $3.00 * 1$ & $3.00 * 1$ & $18,19,27,30,48$ \\
\hline Acer spicatum & & & $2.00+1$ & & 4 \\
\hline Alnus tenuifolia $_{l}$ & $1.00-1$ & & $1.50+2$ & $2.00+1$ & $5,18,48$ \\
\hline Amelanchier alnifolia & $2.50 * 6$ & $2.00+2$ & $2.50 * 2$ & $2.33 * 3$ & $1,4,5,18,19,26,27,30,42,48$ \\
\hline Arctostaphylos uva-ursi & $1.00-2$ & $1.00-1$ & & $1.50+2$ & $4,29,42$ \\
\hline Artemisia cana & $2.00+1$ & & & & 32 \\
\hline Artemisia tridentata & $1.50+8$ & $1.25-4$ & $1.00-2$ & $1.67+3$ & $5,6,13,19,21,22,25,27,32,37,39,43,46$ \\
\hline Artemisia tripartita & $3.00 * 1$ & $2.00+1$ & & & 39 \\
\hline Berberis repens & $2.00+2$ & $1.25-4$ & $1.00-1$ & $1.80+10$ & $1,2,6,21,23,24,25,28,29,30,36,39,45$ \\
\hline Betula glandulosa & $2.00+2$ & & $3.00 * 1$ & & 18,30 \\
\hline Betula fontinalis & $1.00-1$ & & & & 18 \\
\hline Ceanothus velutinus & $2.40 * 5$ & & $3.00 * 1$ & $2.00+2$ & $13,18,19,30,33,47,48$ \\
\hline Ceanothus sanguineus & $3.00 * 3$ & & $3.00 * 1$ & $3.00 * 1$ & $18,45,47,48$ \\
\hline Cercocarpusmontanus & $3.00 * 2$ & & & & 27,31 \\
\hline Cercocarpus ledifolius & $2.00+1$ & & & $3.00 * 1$ & 1,13 \\
\hline Chrysothamus nauseosus & $2.00+3$ & $1.00-1$ & & & $12,13,43,46$ \\
\hline Chrysothamus viscidiflorus & $1.25-4$ & $3.00 * 1$ & & $2.00+2$ & $12,20,32,43,46$ \\
\hline Cornus stolonifera & $2.20+5$ & & $3.00 * 1$ & $2.00+1$ & $4,18,19,27,45,48$ \\
\hline Dasiphora fruticosa & $1.00-1$ & & & & 18 \\
\hline Elaeagnus commutata & $2.50 * 2$ & & & & 18,30 \\
\hline Fraxinus & $2.00+1$ & & & & 26 \\
\hline Garrya wrightii & $3.00 * 1$ & & & & 31 \\
\hline Juniperus & $1.50+2$ & & & $1.00-1$ & 24,31 \\
\hline Juniperus communis & $1.00-3$ & & & $1.00-1$ & $18,29,42$ \\
\hline Juniperus horizontalis & $1.00-3$ & & & & $11,30,42$ \\
\hline Juniperus occidentalis & $2.00+1$ & & & & 13 \\
\hline Juniperus scopulorum & $1.50+2$ & $1.00-1$ & & $1.00-1$ & 18,32 \\
\hline Ledum groenlandicum & $2.00+1$ & & & & 4 \\
\hline Linnaea & & $1.00 \quad 1$ & & $1.00 \quad 1$ & 29 \\
\hline Linnaea borealis & $1.00-1$ & & & & 18 \\
\hline Lonicera involucrata & $2.00+1$ & & $2.00+1$ & & 18,48 \\
\hline Lonicera utahensis & & & $3.00 * 1$ & $1.00-1$ & 48 \\
\hline Menziesia ferruginea & & & $3.00 * 1$ & & 48 \\
\hline Odostemon sp. & $1.00-1$ & & & & 13 \\
\hline Odostemon aquifolium & $1.00-1$ & & & & 18 \\
\hline
\end{tabular}




\begin{tabular}{|c|c|c|c|c|c|}
\hline Opulaster malvaceus & $1.00-1$ & & $2.00+1$ & $1.00-1$ & 48 \\
\hline Pachist ima myrsinites & $1.50+2$ & & & $2.33 * 3$ & $2,13,18,28,31$ \\
\hline Philadelphus lewisii & $3.00 * 1$ & & & & 18 \\
\hline Pinus & $1.00-3$ & & & $1.00-1$ & $6,24,39,45$ \\
\hline Pinus conterta & $1.33-3$ & & & $1.50+2$ & $18,21,25,30,42$ \\
\hline Pinus flexis & & & & $1.00-1$ & 36 \\
\hline Pinus ponderosa & 1.002 & & & & 13,18 \\
\hline Populus balsamifera & $2.00+1$ & & & & 4 \\
\hline Populus tremuloides & $2.50 * 8$ & $2.25 * 4$ & $1.74+4$ & $2.50 * 8$ & $2,4,5,6,9,18,25,27,30,31,36,42,48$ \\
\hline Populus trichocarpa & $3.00 * 1$ & & & & 18 \\
\hline Potentilla sp. & & & & $1.00-1$ & 9 \\
\hline Potentilla fruticosa & $1.00-1$ & & & & 30 \\
\hline Prunus virginiana & $2.38 * 8$ & $2.00+3$ & $2.00+2$ & $1.50+2$ & $4,12,18,21,26,27,30,32,39,42,46,48$ \\
\hline Prunus emarginata & & & $3.00 * 1$ & $3.00 * 1$ & 48 \\
\hline Prunus pensylvanica & $2.00+1$ & & & & 4 \\
\hline Pseudotsuga menziesii var.glauca & $1.27-11$ & $1.00-3$ & & $2.00+1$ & $5,6,13,15,18,21,24,25,29,32,42,45$ \\
\hline Purshia tridentata & $3.00 * 4$ & & & $3.00 * 1$ & $2,12,13,27,46$ \\
\hline Quercus gambellii & $3.00 * 3$ & $2.00+1$ & & & $5,26,27$ \\
\hline Rhamnus purshiana & & & $1.00-1$ & & 48 \\
\hline Ribes & $1.00-1$ & & & & 18 \\
\hline Ribes cereum & & & $1.00-1$ & & 5 \\
\hline Ribes cognatum & & & $3.00 * 1$ & $2.00+1$ & 48 \\
\hline Ribes montigenum & & & $3.00 * 1$ & & 37 \\
\hline Ribes petiolare & & & $1.00-1$ & $1.00-1$ & 48 \\
\hline Ribes viscosissimum & & & $3.00 * 1$ & $2.00+1$ & 48 \\
\hline Rosa & $1.50+2$ & & $3.00 * 1$ & $2.00 * 1$ & $18,42,48$ \\
\hline Rosa acicularis & $1.00-2$ & & $3.00 * 1$ & $3.00 * 1$ & 4,38 \\
\hline Rubus & & & & $1.00-1$ & 25 \\
\hline Rubus idaeus & & & $2.00+1$ & & 4 \\
\hline Rubus parviflorus & & & $3.00 * 1$ & $2.00+1$ & 48 \\
\hline Salix & $2.11+9$ & $2.00+1$ & $1.71+7$ & $2.29 * 7$ & $4,6,13,19,21,22,25,29,30,33,3639,42,44,47,48$ \\
\hline Salix bebbiana & $3.00 * 1$ & & & & 18 \\
\hline Salix exigua & $2.00+1$ & & & & 18 \\
\hline Salix geyeriana & $2.00+1$ & & & & 18 \\
\hline Salix lutea & $3.00 * 1$ & & & & 18 \\
\hline Salix melanopsis & $2.00+1$ & & & & 18 \\
\hline Salix scouleriana & $1.50+2$ & & & & 18,45 \\
\hline Salix subcoerulea & $3.00 * 1$ & & & & 18 \\
\hline Sambucus sp. & & & $3.00 * 1$ & & 3 \\
\hline Sambucus caerulea & & & $3.00 * 1$ & $3.00 * 1$ & 48 \\
\hline Sambucus melanocarpa & $3.00 * 1$ & & $3.00 * 1$ & $3.00 * 1$ & 18,48 \\
\hline Sericotheca discolor & $1.00-1$ & & $2.00+1$ & $1.00-1$ & 47,48 \\
\hline Shepherdia canadensis & $2.00+1$ & & $1.00-1$ & $2.00+1$ & $25,33,42$ \\
\hline Sorbus americana & & & $3.00 * 1$ & $3.00 * 1$ & 48 \\
\hline Sorbus occidentalis & $2.00+1$ & & & & 18 \\
\hline Spirea & $1.00-1$ & & $1.00-1$ & $1.00-1$ & 18,29 \\
\hline Spirea betulifolia & $2.00+1$ & & & & 42 \\
\hline Spirea lucida & & & $2.00+1$ & $2.00+1$ & 48 \\
\hline Spirea menziesii & & & $2.00+1$ & $2.00+1$ & 48 \\
\hline Symphoricarpos & $1.40-5$ & & $1.50+2$ & $1.83+6$ & $13,23,25,27,29,32,33,36,42,47$ \\
\hline Symphoricarpos albus & $3.00 * 1$ & & $3.00 * 1$ & $2.00+2$ & $4,18,48$ \\
\hline Symphoricarpos occidentalis & $1.00-1$ & & & & 39 \\
\hline Symphoricarpos utahensis & & $1.00-1$ & $1.00-1$ & & 5 \\
\hline Tetradymia canescens & $1.50+2$ & & & $1.50+2$ & 46,39 \\
\hline Thuja plicata & $2.00+1$ & & & & 45 \\
\hline Vaccinium & $2.00+2$ & & $1.00-3$ & $1.00-2$ & $6,13,25,30,36,44$ \\
\hline Vaccinium membranaceum & $1.00-1$ & & $3.00 * 1$ & $1.33-3$ & $18,29,33,48$ \\
\hline Vaccinium scoparium & $1.00-1$ & & $1.00-1$ & $1.00-3$ & $9,18,29,31,48$ \\
\hline
\end{tabular}

Forbs having the best documented highly valuable rankings as summer forage were Agoseris glauca and Geranium viscossimum. Lupinus spp. was rated as highly valuable fall forage on the basis of five studies. Aster spp. was frequently mentioned and was considered a valuable plant throughout the year.

Highly valuable grasses or grasslikes having the best documented rankings were Agropyron spicatum, Carex sp.,
Carex geyeri, Festuca idahoensis, Festuca scabrella, and Poa sp. Highly valuable ratings were seasonal for most of these species. Only Carex geyeri was rated highly valuable throughout the year. Koeleria cristata was well documented as a valuable species on an annual basis.

Among shrubs, the highly valuable species with rankings based on a relatively large number of references were Amelanchier alnifolia, Ceanothus sanguineus,
Ceanothus velutinus, Populus tremuloides, Prunus virginiana, Purshia tridentata, Quercus gambellii and Salix spp. Like forbs and grasses, these shrubs were highly valuable during only certain portions of the year. Most were highly valuable during the fall or winter.

\section{Discussion}

The validity of some value rankings could be influenced by the fact that 
various strains of the same species can differ in palatability. Palatability may also vary with differences in climate, soil conditions, and topography. However, the impact of these factors on rankings cannot be assessed until their effects on palatability of all elk forage species have been researched extensively.

Relative abundance and availability are two other factors which may have influenced value rankings. Some species may have received least valuable rankings because they were not abundant or because they were relatively unavailable due to some factor such as snow cover. Thus, they were only minor contributors to the diet. They may be highly valuable when they are more abundant or available. However, unless the normal abundance or availability of such species can be increased through management, their assigned rankings must stand as indications of their value as elk forage under natural conditions.

It must be remembered that rankings contained herein are averages for all studies where the species were eaten. Thus, some elk managers working where food habits have been studied extensively may feel that certain ratings are too high or low for their particular area. In such instances, questionable rankings may be adjusted up or down to fit the circumstances. However, the real benefits from these rankings should be realized by managers who lack sufficient data to determine the relative forage value of plants in their area and by managers who want to revegetate their ranges with plant species known to be good elk forage.

\section{Literature Cited}

1. Anderson, Chester C. 1958. Reconnaissance of deer habitat in special problem areas. Wyo. Fish and Game Comm. Fed. Aid Compl. Rep. Proj. W-27-R11, WP-4, J-7 and 8.29 p.

2. Anderson, C. C., W. I. Crump, and T. C. Baker. 1956. Food habits of antelope, elk, deer and moose. Wyo. Game and Fish Comm. Fed. Aid Compl. Rep. Proj. W-27-R9, WP-1, J-1 . p. 8-31.

3. Baker, Theodore C., Chester Anderson, and William 1. Crump. 1953. Food habits study of game animals. Wyo. Wild. $17(11): 24-31$.

4. Blood, Donald A. 1966. Range relationships of elk and cattle in Riding Mountain National Park, Manitoba. Canadian Wildl. Serv. Wildl. Manage. Bull., Scries 1, No. 19. $62 \mathrm{p}$.

5. Boyd, Raymond J. 1970. Elk of the White River Plateau, Colorado. Colo. Div. Game, Fish and Parks. Tech. Bull. No. 25. 126 p.

6. Boyd, Raymond J.1972. Stomach contents data from the Rio Grande elk herd in southwestern Colorado. Colo. Div. Game, Fish and Parks, Fort Collins, (Unpublished).
7. Brazda, A. R. 1953. Elk migration patterns and some of the factors affecting movements in the Gallatin River drainage, Montana. J. Wild1. Manage. 17:9-23.

8. Buechner, Helmut K. 1952. Winter-range utilization by elk and mule deer in southeastern Washington, J. Range Manage. $5: 76-80$.

9. Burt, Theo. Jr., and Gerald H. Gates. 1959. Pecos elk reproductive and food habits studies. New Mex. Dep. of Game and Fish. Fed. Aid Compl. Rep. Proj. W-93-R-1, WP-3, J-6. $19 \mathrm{p}$.

10. Capp, John C. 1967. Competition among bighorn sheep, elk and deer in Rocky Mountain National Park, Colorado. M.S. Thesis. Colo. State Univ., Fort Collins. 132 p.

11. Casgranda, Lloyd, and Reuel G. Janson. 1957. Wildlife investigations (Dist. 4): Big Game Surveys and Investigations. Montana Dep. of Fish and Game. Fed. Aid Compl. Rep. Proj. W-74-R-2, J-A1, Pt. 3. 19 p.

12. Chadwick, Howard William. 1960. Plant succession and big game winter movements and feeding habits in a sand dune area in Fremont County, Idaho. M.S. Thesis. Univ. of Idaho, Moscow. $121 \mathrm{p}$.

13. Cliff, Edward P. 1939. Relationship between elk and mule deer in the Blue Mountains of Oregon. Trans. N. Am. Wildl. Conf. 4:560-569.

14. Constan, Kerry John. 1967. Bighorn sheep range use, food habits and relationships to mule deer and elk in Gallatin Canyon. M.S. Thesis, Montana State Univ., Bozeman. 43 p.

15. Cowan, I. MC T. 1947. Range competition between mule deer, bighorn sheep and elk in Jasper Park, Alberta. Trans N. Amer. Wildl. Conf. 12:223-227.

16. De Nio, R. M. 1938. Elk and deer foods and feeding habits. Trans N. Amer. Wildl. Conf. 3:421-427.

17. Eustace, C. D. 1967. Food habits, range use and relationships between elk and livestock in the Gravelly Mountains, Montana. M.S. Thesis. Montana State Univ., Bozeman. $55 \mathrm{p}$.

18. Gaffney, W. D. 1941. The effects of winter elk browsing, south fork of the Flathead River, Montana. J. Wildl. Manage. 5:427.

19. Geis, A. F. 1954. The food requirements and relative digestibility of a variety of winter diets fed to elk (Cerrus canadensis nelsoni) under controlled conditions. M.S. Thesis. Montana State Univ., Missoula. 68 p. 20. Gordon, Floyd A. 1968. Range relationships of elk and cattle on elk winter range, Crow Creek, Montana. M.S. Thesis. Montana State Univ., Bozeman. $52 \mathrm{p}$.

21. Greer, Kenneth R. 1959. Analysis of 68 samples from the 1956 Gallatin Canyon elk "Hunter kill." In Wildlife investigationsState: Wildlife investigations laboratory. Montana Dep. of Fish and Game. Fed. Aid Compl. Rep. Project W-83-R-2. All jobs. p. 5-6.

22. Greer, Kenneth R. 1959. Analysis of 23 elk rumen samples collected during 1957 from the vicinity of Gardiner, Montana. In Wildlife investigations-State: Wildlife investigations laboratory. Montana Dep. of Fish and Game. Fed. Aid Compl. Rep. Proj. W-83-R-2. All jobs. p. 8.

23. Greer, Kenneth R. 1960. Analysis of twenty-one elk rumens collected from the 1956 hunter-kill at Boyd Ranch, Missoula County. In Wildlife investigations-State:
Wildlife investigations laboratory. Montana Dep. of Fish and Game. Fed. Aid Compl. Rep. Proj. W-83-R-3. All jobs. p. 14.

24. Greer, Kenneth R. 1960. Analysis of twenty-nine elk rumens collected during 1956-57 from the Sun River Game RangeLewis and Clark County, Montana. In Wildlife investigations-State: Wildlife investigations laboratory. Montana Dep. of Fish and Game. Fed. Aid Compl. Rep., Proj. W-83-R3. All jobs. p. 16-17.

25. Greer, Kenneth R., John B. Kirsch, and Harley W. Yeagher. 1970. Seasonal food habits of the northern Yellowstone elk (Wapiti) herds during 1957 and 1962-1967 as determined from 793 rumen samples. Montana Dep. of Fish and Game. Fed. Aid Final Rep. Proj. W-83-R-12, J-B-1. 76 p.

26. Greffenius, R. J. 1938. Results of the Copper Ridge Basin elk study. U. S. Forest Serv., Rocky Mountain Region. Bull. 21(4):14-15.

27. Harris, John T. 1958. Analysis of elk winter range, south fork of the White River, Colorado. M.S. Thesis. Colo. State Univ., Fort Collins. 136 p.

28. Hoskins, Leonard Wayne. 1952. Browse utilization studies on the Pocatello winter big game range. M.S. Thesis. Univ. of Idaho, Moscow. 87 p.

29. Kirsch, John B. 1963. Range use, relationships to logging, and food habits of the elk in the Little Belt Mountains, Montana. M.S. Thesis. Montana State Univ., Bozeman. 44 p.

30. Knight, Richard R. 1970. The Sun River elk herd. Wildl. Monogr. No. 23. 66 p.

31. Lang, E. M. 1958. Elk of New Mexico. New Mexico Dep. of Game and Fish. Bull. No. 8. $33 \mathrm{p}$.

32. Mackie, Richard J. 1970. Range ecology and relations of mule deer, elk and cattle in the Missouri River Breaks, Montana. Wildl. Monogr. No. 20. 79 p.

33. Martinka, C. J. 1969. Population ecology of summer resident elk in Jackson Hole, Wyoming. J. Wildl. Manage. 33:465-481.

34. Morris, M. S., and J. E. Schwartz. 1957. Mule deer and elk food habits on the National Bison Range. J. Wildl. Manage. 21:189193.

35. Nichols, Lyman, Jr. 1957. Forage utilization by elk and domestic sheep in the White River National Forest. M.S. Thesis. Colo. State Univ., Fort Collins. 92 p.

36. Peek, James M. 1963. Wildlife investigations (Dist. 3): Big game survey and investigation-summer of 1956-1960, GravellySnowcrest rumen collections. Montana Dept. of Fish and Game. Fed. Aid Compl. Rep. Proj. W-73-R-8, J-A-1. 11 p.

37. Pickford, G. D., and Elbert H. Reid. 1943. Competition of elk and domestic livestock for summer range forage. J. Wildl. Manage. 7:328-332.

38. Picton, Harold D. 1960. A comparison of the results of feeding site examinations with the vegetative composition of the sites in the Sun River Game Range. In Wildlife investigations (Dist. 4): Big game surveys and investigations. Montana Dep. Fish and Game. Fed. Aid Compl. Rep. Proj. W-74-R-5, J-A-1. p. 9.

39. Rouse, R. A. 1957. Elk food habits, range use and movements. Gravelly Mountains, Montana. M.S. Thesis, Montana State Univ., Bozeman. 29 p.

40. Rouse, R. A. 1958. Wildlife investigations 
(Dist. 3): Elk investigations (elk-livestock relationships). Montana Dep. of Fish and Game. Fed. Aid Compl. Rep. Proj. W-73-R-3, J-A-2. 3 p.

41. Rush, W. M. 1932. Northern Yellowstone elk study. Montana Fish and Game Comm., Helena. $131 \mathrm{p}$.

42. Schallenberger, Allen. 1965. Big game forage competition in Sun River Canyon. M.S.

Thesis. Montana State Univ., Bozeman. 43 p.

43. Snyder, Roy. 1969. Forage competition between cattle and elk in the Gird Creek drainage of western Montana. M.S. Thesis. Univ. of Montana, Missoula. 86 p.

44. Stevens, David R. 1966. Range relationships of elk and livestock, Crow Creek drainage, Montana. J. Wildl. Manage. 30:349-363.

45. Trout, Lester C., and Thomas A. Leege. 1971. Are the northern Idaho elk herds doomed? Idaho Wildl. Rev. 24(3):3-6.

46. Wing, Larry Dean. 1962. Big game and livestock browse utilization and feeding habits on a sandy range in southeastern Idaho. M.S. Thesis. Univ. of Idaho, Moscow. $89 \mathrm{p}$.

47. Young, Vernon A. 1938. The carrying capacity of big game range. J. Wildl. Manage. 2:131-134.

48. Young, Vernon A., and W. Leslie Robinette. 1939. A study of the range habits of elk on the Selway Game Preserve. Univ. of Idaho, School of Forestry, Bull. No. 9.47 p. 